\title{
ON SUBHARMONIC FUNCTIONS
}

\author{
MAXWELL READE
}

If $p(x, y)$ is continuous in a domain (non-null connected open set) $\mathcal{D}$, then $p(x, y)$ is subharmonic in $\mathcal{D}$ if and only if the inequality

$$
p(x, y) \leqq A(p ; x, y ; r) \equiv \frac{1}{\pi r^{2}} \iint_{D(x, y ; r)} p(x+\xi, y+\eta) d \xi d \eta
$$

holds for all circular discs $D\left(x_{0}, y_{0} ; r\right):\left(x-x_{0}\right)^{2}+\left(y-y_{0}\right)^{2}=\xi^{2}+\eta^{2} \leqq r^{2}$ in $\mathcal{D}$. If $p(x, y)$ is continuous along with its partial derivatives of the second order in $\mathcal{D}$, then $p(\dot{x}, y)$ is subharmonic there if and only if $\Delta p(x, y) \geqq 0$ in $\mathcal{D}$, where $\Delta$ is the Laplace operator $[2,3] .{ }^{1}$

If $p(x, y)$ is continuous in $\mathcal{D}$, then $p(x, y)$ is said to be of class $P L[2]$ in $\mathcal{D}$ provided (i) $p(x, y) \geqq 0$ and (ii) $\log p(x, y)$ is subharmonic wherever $p(x, y) \neq 0$. If $p(x, y) \geqq 0$ and is continuous along with its partial derivatives of the second order, then $p(x, y)$ is of class $P L$ if and only if $p \Delta p-p_{x}^{2}-p_{y}^{2} \geqq 0$ wherever $p(x, y) \neq 0$.

Beckenbach [1] has proved the following theorem characterizing functions of class $P L$.

Theorem A. If $p(x, y) \geqq 0$ in $\mathcal{D}$, then $p(x, y)$ is of class $P L$ in $\mathcal{D}$ if and only if $\left[(x-\alpha)^{2}+(y-\beta)^{2}\right] p(x, y)$ is subharmonic in $\mathcal{D}$ for every choice of the real constants $\alpha, \beta$.

The Beckenbach theorem is comparable to the classic MontelRadó theorem, which was later generalized by Kierst and Saks [3, 4] for functions $p(x, y)$ with continuous partial derivatives of the second order; this generalization is the following theorem.

TheOREM B. Let $f(t)$ have a continuous second derivative, with $f^{\prime}(t)>0$, for $-\infty<t<\infty$. If $v(x, y)$ has continuous partial derivatives of the second order in $\mathcal{D}$, and if the function $f(\alpha x+\beta y+v(x, y))$ is subharmonic in $\mathcal{D}$ for every choice of the real constants $\alpha, \beta$, then $v(x, y)$ is subharmonic in $\mathcal{D}$.

The question arises as to the possibility of exhibiting a Kierst-Saks type of generalization for Beckenbach's Theorem A. Our result is the following.

Presented to the Society, November 27, 1942, under the title Remarks on a paper of Bechenbach; received by the editors February 26, 1943.

${ }^{1}$ The numbers in square brackets refer to references listed in bibliography at end of paper. 
THEOREM C. Let $f(t)$ have a continuous second derivative, with $f^{\prime}(t)>0$, for $-\infty<t<\infty$. If $v(x, y)$ has continuous partial derivatives of the second order in $\mathcal{D}$, and if the function

$$
\Phi(v ; \alpha, \beta ; x, y) \equiv f\left\{\log \left[(x-\alpha)^{2}+(y-\beta)^{2}\right]+v(x, y)\right\}
$$

is subharmonic in $\mathcal{D}$ for every choice of the real constants $\alpha, \beta$, then $v(x, y)$ is subharmonic in $\mathcal{D}$.

PRoof. We shall show $\Delta v(x, y) \geqq 0$. From the hypothesis, it follows that $\Phi(v ; \alpha, \beta ; x, y)$ has a non-negative Laplacian, that is,

$$
\begin{aligned}
f^{\prime}(t) \Delta v(x, y)+f^{\prime \prime}(t) & \left\{\left[\frac{2(x-\alpha)}{(x-\alpha)^{2}+(y-\beta)^{2}}+v_{x}(x, y)\right]^{2}\right. \\
+ & {\left.\left[\frac{2(y-\beta)}{(x-\alpha)^{2}+(y-\beta)^{2}}+v_{y}(x, y)\right]^{2}\right\} \geqq 0, }
\end{aligned}
$$

where $t \equiv \log \left[(x-\alpha)^{2}+(y-\beta)^{2}\right]+v(x, y)$, for all real $\alpha, \beta$.

Let $\left(x_{0}, y_{0}\right)$ be a point of $\mathcal{D}$. We distinguish two cases. (a) $v_{x}^{2}+v_{y}^{2}$ $\neq 0$ at $\left(x_{0}, y_{0}\right)$. Then it is sufficient to choose

$$
\alpha=x_{0}+2 v_{x} /\left(v_{x}^{2}+v_{y}^{2}\right), \quad \beta=y_{0}+2 v_{y} /\left(v_{x}^{2}+v_{y}^{2}\right),
$$

where the partial derivatives are evaluated at $\left(x_{0}, y_{0}\right)$, and then to make use of the positiveness of $f^{\prime}(t)$ to obtain $\Delta v\left(x_{0}, y_{0}\right) \geqq 0$ from (2). (b) $v_{x}^{2}+v_{y}^{2}=0$ at $\left(x_{0}, y_{0}\right)$. If $\left(x_{0}, y_{0}\right)$ can be approached by a sequence of points $\left(x_{n}, y_{n}\right), n=1,2, \cdots$, at each of which $v_{x}^{2}+v_{y}^{2} \neq 0$, then it follows from (a) and the continuity of $\Delta v(x, y)$ that $\Delta v\left(x_{n}, y_{n}\right)$ $\rightarrow \Delta v\left(x_{0}, y_{0}\right) \geqq 0$, as $n \rightarrow \infty$. If no such sequence exists, then $v_{x}^{2}+v_{y}^{2} \equiv 0$ in a neighborhood of $\left(x_{0}, y_{0}\right)$, so that $\Delta v\left(x_{0}, y_{0}\right)=0$. This completes the proof.

Beckenbach has also given an isoperimetric characterization of functions of class $P L[2]$; if we use the abbreviation

$$
L(p ; x, y ; r) \equiv \frac{1}{2 \pi r} \int_{C(x, y ; r)} p(x+\xi, y+\eta) d s
$$

where $C(x, y ; r)$ is the boundary of $D(x, y ; r)$, then the theorem is the following.

ThEorem D. If $p(x, y)$ is continuous and if $p(x, y) \geqq 0$ in $\mathcal{D}$, then $p(x, y)$ is of class $P L$ in $D$ if and only if, for every continuous function $q(x, y)$ of class $P L$ in $\mathcal{D}$, the inequality

$$
A(p q ; x, y ; r) \leqq L(p ; x, y ; r) \cdot L(q ; x, y ; r),
$$

holds for every circular disc $D(x, y ; r)$ in $\mathcal{D}$. 
This latter theorem may be extended as follows, for functions that have continuous second derivatives in $\mathcal{D}$.

THEOREM E. If $p(x, y)$ has continuous partial derivatives of the second order in $\mathcal{D}$, if $p(x, y) \geqq 0$ in $\mathcal{D}$, and if $a, b$ is a pair of real constants such that $(1 / a)+(1 / b)=2$, then $p(x, y)$ is of class PL in $\mathcal{D}$ if and only if, for every continuous function $q(x, y)$ of class $P L$ in $D$, the inequality

$$
A(p q ; x, y ; r) \leqq\left[L\left(p^{a} ; x, y, r\right)\right]^{1 / a} \cdot\left[L\left(q^{b} ; x, y ; r\right)\right]^{1 / b}
$$

holds for every circular disc $D(x, y ; r)$ in $\mathcal{D}$.

Necessity. A proof of the necessity part may be given that parallels Beckenbach's proof of his theorem; indeed, the only change would be to use the more general Hölder inequality where Beckenbach uses the Schwarz inequality. It is to be noted that the hypothesis on the existence of any derivative of $p(x, y)$ is superfluous here.

Sufficiency. Let $\left(x_{0}, y_{0}\right)$ be a point of $\mathcal{D}$ such that $p\left(x_{0}, y_{0}\right) \neq 0$; if there were no such point, then $p(x, y) \equiv 0$ and hence of class $P L$ in $\mathcal{D}$. We shall consider only analytic $q(x, y)$, so that we may expand both $p(x, y)$ and $q(x, y)$ in finite Taylor expansions about $\left(x_{0}, y_{0}\right)$, and then substitute in (3) to obtain

$$
\begin{aligned}
& p q+\left(r^{2} / 8\right) \Delta(p q) \\
& \quad \leqq\left[p^{a}+\left(r^{2} / 4\right) \Delta\left(p^{a}\right)+o\left(r^{2}\right)\right]^{1 / a}\left[q^{b}+\left(r^{2} / 4\right) \Delta\left(q^{b}\right)+o\left(r^{2}\right)\right]^{1 / b}+o\left(r^{2}\right),
\end{aligned}
$$

where $o\left(r^{2}\right)$ is a quantity (not always the same quantity) such that $\left[o\left(r^{2}\right) / r^{2}\right] \rightarrow 0$, as $r \rightarrow 0$. We use binomial expansions, for small $r$, and then let $r \rightarrow 0$, to obtain the further inequality

$$
\Delta(p q) / 8 \leqq\left(p q / 4 a p^{a}\right) \Delta\left(p^{a}\right)+\left(p q / 4 b q^{b}\right) \Delta\left(q^{b}\right),
$$

where the derivatives are evaluated at $\left(x_{0}, y_{0}\right)$. If we express the derivatives of $p q$, $p^{a}$ and $q^{b}$ in terms of the derivatives of $p$ and $q$, and if we set $q \equiv e^{\alpha x+\beta y}$, we obtain

$$
\begin{aligned}
0 \leqq & (2 b-1)\left(\alpha^{2}+\beta^{2}\right) p^{2}-2 \alpha p p_{x} \\
& -2 \beta p p_{y}+p \Delta p+(2 a-2)\left(p_{x}^{2}+p_{y}^{2}\right),
\end{aligned}
$$

which must hold for all real constants $\alpha, \beta$. Hence the discriminant of the right-hand member of (5) must satisfy

$$
0 \leqq(2 b-1)^{2}\left[p \Delta p+(2 a-2)\left(p_{x}^{2}+p_{y}^{2}\right)\right]-(2 b-1)\left(p_{x}^{2}+p_{y}^{2}\right),
$$

which yields, since $2 b-1>0$ and $(1 / a)+(1 / b)=2$,

$$
0 \leqq p \Delta p-p_{x}^{2}-p_{y}^{2} \text {. }
$$

Hence $p(x, y)$ is of class $P L$ in $\mathcal{D}$. 
The usual averaging process does not appear to be adaptable to the lessening of differentiability conditions in Theorems B, C and E.

\section{BIBLIOGRAPHY}

1. E. F. Beckenbach, Functions having subharmonic logarithms, Duke Math. J. vol. 8 (1941) pp. 393-400.

2. E. F. Beckenbach and T. Rad6, Subharmonic functions and minimal surfaces and Subharmonic functions and surfaces of negative curvature, Trans. Amer. Math. Soc. vol. 35 (1933) pp. 648-661 and pp. 662-674, respectively.

3. T. Rad6, Subharmonic functions, Berlin, 1937.

4. S. Saks, On subharmonic functions, Acta Univ. Szeged. vol. 5 (1930-1932) pp. 187-193.

Purdue University 